\title{
Física estatística aplicada à neurociência de estados alterados: o cérebro sob influência de psicodélicos
}

Statistical physics applied to the neuroscience of altered states: the brain under the influence of psychedelics

\author{
A. Viol ${ }^{* 1}$, H. Felippe Jr. ${ }^{2}$, Fernanda Palhano-Fontes ${ }^{3}$, Heloisa Onias ${ }^{3}$, \\ Draulio B. de Araujo ${ }^{3}$, G. M. Viswanathan ${ }^{2,4}$ \\ ${ }^{1}$ Cognitive Neuroscience, Scuola Internazionale Superiore di Studi Avanzati - SISSA, 34136, Trieste, Italy. \\ ${ }^{2}$ Universidade Federal do Rio Grande do Norte, Departamento de Física, 59078-970, Natal, RN, Brasil. \\ ${ }^{3}$ Universidade Federal do Rio Grande do Norte, Instituto do Cérebro, 59078-970, Natal, RN, Brasil. \\ ${ }^{4}$ Universidade Federal do Rio Grande do Norte, Instituto Nacional de Ciência e Tecnologia de Sistemas Complexos, \\ 59078-970, Natal, RN, Brasil.
}

Received on October 15, 2020. Revised on December 14, 2020. Accepted on December 17, 2020.

\begin{abstract}
O objetivo principal da mecânica estatística é relacionar a física microscópica regida pelas leis de Newton, ou então pela mecânica quântica, com a física macroscópica regida pelas leis da termodinâmica. Conceitos macroscópicos, tais como a temperatura, que não fazem sentido do ponto de vista microscópico, surgem na mecânica estatística como propriedades emergentes quando o número de partículas torna-se suficientemente grande. O sucesso da mecânica estatística em explicar e prever uma variedade de fenômenos macroscópicos motivou a aplicação de conceitos e métodos da física estatística ao estudo de sistemas complexos e redes complexas, inclusive em neurociência. Nosso objetivo neste trabalho é aplicar essa abordagem a um problema chave na neurociência: como entender quantitativamente os correlatos neurais de estados mentais alterados de consciência induzidos por substâncias psicodélicas? Vamos primeiro introduzir alguns conceitos básicos sobre a conectividade funcional das regiões do cérebro. Discutimos então a fenomenologia e a neurociência relacionadas aos psicodélicos. Finalmente, resumimos alguns estudos que aplicaram a física estatística e a teoria de redes complexas para tentar melhor entender o comportamento do cérebro sob influência dos psicodélicos.
\end{abstract}

Palavras-chave: Redes complexas, sistemas complexos, neurociência, psicodélicos.

The primary aim of statistical mechanics is to relate the micropscopic physics governed by Newton's laws, or else by quantum mechanics, to the macroscopic physics governed by the laws of thermodynamics. Macroscopic properties, such as temperature, may have no meaning at the microscopic level, but they arise as emergent properties in statistical mechanics when the number of particles becomes sufficiently large. The success of statistical mechanics in explaining and predicting a variety of macroscopic phenomena has motivated the application of concepts and methods of statistical physics to study complex systems and complex networks, including in neuroscience. Our aim here is to apply this approach to a key problem in neuroscience: how can we quantitatively understand the neural correlates of altered mental states induced by psychedelic substances? We first give an overview of basic concepts concerning functional connectivity of brain regions. We then discuss the phenomenology and the neuroscience related to psychedelics. Finally, we review some studies that have applied statistical physics and the theory of complex networks to try to gain a better understanding of the brain under the influence of psychedelics.

Keywords: Complex networks, complex systems, neuroscience, psychedelics.

\section{Introdução}

O cérebro não é um sistema simples, mas sim um sistema complexo [1 3]. Podemos chamar de "sistemas simples" aqueles que, em princípio, são relativamente fáceis de entender. Um microprocessador de computador, por exemplo, pode ser um sistema "complicado", mas não é um sistema complexo: seu comportamento pode ser compreendido em termos do comportamento de suas partes. Portanto, o que diferencia os siste-

\footnotetext{
*Endereço de correspondência: aline.viol@sissa.it
}

mas complexos dos sistemas simples é a dificuldade em modelar o comportamento do último. Aqui, não definiremos formalmente o que é um sistema complexo, mas tão somente listaremos algumas de suas propriedades: (i) organização hierárquica; (ii) dinâmica ou relacionamentos não lineares entre seus subsistemas; (iii) auto-organização; (iv) propriedades emergentes; e (v) memória ou correlações, possivelmente de longo alcance. Veremos que o cérebro possui todas essas características.

As técnicas e abordagens que tradicionalmente são usadas para descrever quantitativamente os sistemas simples nem sempre são aplicáveis aos sistemas 
complexos. Por exemplo, os métodos matemáticos para solucionar equações diferenciais lineares, mesmos os de alta ordem, tipicamente falham quando aplicados à dinâmica não linear. Até mesmo a quantificação correta da complexidade se apresenta como um problema, pois não é uma medição intuitiva. Tomemos, como exemplo, a caracterização da complexidade de uma sequência binária $s$ finita. A complexidade de Kolmogorov de $s$ é definida como sendo o menor tamanho (em bits) de todas as possíveis representações ou descrições de $s$. Ou seja, a complexidade de Kolmogorov é o tamanho do menor algoritmo que reproduz o objeto.

A título de ilustração, considere a sequência $12121212 \ldots$. (até completarmos cem algarismos). Note que, embora longa, a sequência pode ser "comprimida" usando o simples algoritmo "repita o número 12 cinquenta vezes". Por se tratar de um algoritmo menor que a sequência original, dizemos que aquela é de pouca complexidade. $\mathrm{O}$ mesmo não pode ser dito para uma sequência de cem dígitos aleatoriamente escolhidos entre $[0,9]$, pois não há algoritmo com menos de cem algarismos capaz de reproduzi-la, sendo, nesse sentido, mais complexa que a sequência $12121212 \ldots$ Apesar de sua utilidade, a complexidade de Kolmogorov não é uma função computável (por uma máquina de Turing ou similar), o que significa que não existe uma caracterização de complexidade para qualquer $s$ [4. A prova da não computabilidade da complexidade de Kolmogorov é razoavelmente fácil e a recomendamos ao leitor interessado, mas neste trabalho não salientaremos tais aspectos da complexidade.

À dificuldade no tratamento de sistemas complexos, soma-se o reducionismo que procura explicar o comportamento do todo em termos das partes. Podemos ver os limites dessa abordagem quando a aplicamos ao mercado financeiro, onde, embora todas as operações se resumem à compra e venda de ativos, julga-se extremamente difícil modelar o comportamento em larga escala do mercado como um todo. De fato, o mercado financeiro é mais que a coleção de agentes econômicos interagentes, da mesma forma que um cérebro é muito mais que a mera soma de neurônios interligados. Tais considerações deixam claro o grau de dificuldade de estudar os sistemas complexos. As limitações dos métodos tradicionais, por sua vez, motivaram a busca por novas abordagens.

Nessa conjuntura, a física estatística vem contribuindo para o estudo de sistemas complexos há algumas décadas [1, 2, 5,8]. O objetivo original da mecânica estatística consistia em construir a ponte entre a física microscópica regida pelas leis de Newton e a física macroscópica regida pelas leis da termodinâmica [9, 10]. No século XX, o papel das leis de Newton foi substituído pelo da mecânica quântica, mas mesmo com essa mudança a estrutura formal da mecânica estatística sofreu poucas alterações. Várias propriedades macroscópicas, tais como pressão e temperatura, são propriedades emergentes que não estão presentes na escala microscópica dos átomos e partículas individuais que compõem um sistema macroscópico. Tais propriedades são chamadas de emergentes porque "emergem" somente quando o número de partículas torna-se suficientemente grande.

De forma análoga, certas características de sistemas complexos também podem ser abordadas usando os conceitos e métodos da mecânica estatística. Vale a pena destacar que propriedades e fenômenos mentais, tais como consciência, atenção e emoção, aparentam não existir em neurônios individuais. Elas emergem apenas quando os neurônios estão organizados adequadamente e em grande número, na forma que conhecemos de "cérebro". A física estatística naturalmente sugere abordar certas propriedades mentais como sendo propriedades emergentes.

Neste trabalho, nosso objetivo é aplicar essa abordagem a um problema chave na neurociência: como entender quantitativa e qualitativamente os correlatos neurais de estados alterados de consciência em seres humanos, tais como aqueles induzidos por substâncias psicodélicas. A Seção 2 trata da conectividade funcional das regiões do cérebro. As Seções 3 e 4 fazem um breve resumo sobre os psicodélicos e seus efeitos. A Seção 5 discute uma ideia que surgiu na última década, conhecida como "hipótese do cérebro entrópico". A aplicação da física estatística ao estudo de psicodélicos é tratada na Seção6. Finalmente, na Seção 7 encontram-se nossos comentários finais.

\section{A Conectividade Funcional do Cérebro Humano}

Antes de nos concentrarmos em aspectos funcionais e emergentes do cérebro humano, vamos enfatizar as outras características (i)-(v) que o tornam um ilustrativo sistema complexo.

Desde sua anatomia, o cérebro apresenta propriedades complexas por meio de estruturas hierárquicas: sua região mais externa, o córtex cerebral, possui aproximadamente $10^{10}$ neurônios que se auto-organizam em camadas "horizontais" [11, 12]. Os neurônios, por sua vez, operam em regime dinâmico não-linear: em geral, um neurônio dispara somente quando certo limiar de potencial elétrico é alcançado, de tal forma que o princípio de superposição linear não é aplicável. Ademais, as típicas "rugas" do córtex são constituídas por dobras e dobras nas dobras, de tal maneira que o cérebro exibe aspectos de natureza fractal [13. Por fim, a complexidade do cérebro pode ser vista em diferentes escalas: nas interações químicas [14, nas conexões anatômicas [15], comunicação sináptica entre neurônios [16, nas relações funcionais [17], entre outros. Cada um desses tópicos forma, por si, um campo de estudos na neurociência. Focaremos em fornecer ao leitor uma ideia geral sobre a conectividade funcional do cérebro humano em larga escala, isto é, considerando as relações funcionais de diferentes regiões corticais. 
A conectividade funcional indica dependências estatísticas entre sinais. Aqui, é importante ressaltar que não se trata de uma definição de causalidade ou de comunicação entre as partes, mas de coerência temporal da atividade cerebral em diferentes regiões do cérebro. Mas então, o que podemos inferir das redes de conectividade funcional? Para ilustrar essa questão, imagine que a atividade cerebral seja semelhante à performance de uma orquestra auto-organizada. Apesar de não haver um roteiro determinístico, os músicos não tocam aleatoriamente, eles se coordenam em torno de um tema e por meio de estruturas organizacionais. O mapeamento de conectividade funcional nos auxilia a decodificar essas estruturas e tendências implícitas a partir da música.

Desde a década de 1990, o mapeamento da conectividade funcional cerebral avançou consideravelmente, devido, em grande parte, ao aprimoramento de outras técnicas de imageamento cerebral in vivo (como, por exemplo, a encefalografia), e ao surgimento da ressonância magnética funcional (fMRI, do inglês, functional magnetic resonance imaging) 18.

A fMRI é uma técnica que mede, indiretamente, a atividade de populações neuronais através do monitoramento do sinal BOLD (do inglês, blood-oxgenlevel-dependent). A atividade neuronal aumenta a demanda metabólica local, resultando em variações hemodinâmicas e aumento de oxigenação sanguínea, refletida pelo sinal BOLD. A variação na taxa de oxigênio altera a susceptibilidade magnética local devido às propriedades magnéticas da hemoglobina, a proteína do sangue responsável pelo transporte do oxigênio [19]. O mecanismo por detrás do imageamento cerebral através de um escâner de ressonância magnética é baseado em princípios físicos fundamentais e merecem uma leitura cautelosa. Indicamos as referências [19 21 para um estudo mais aprofundado.

A imagem de fMRI pode ser entendida como um filme tridimensional da dinâmica da atividade do cérebro inteiro. A variação da intensidade do sinal de cada voxel (o análogo tridimensional de um pixel) gera uma série temporal. Para mapear a conectividade funcional na escala das regiões corticais, é necessário a extração de uma série temporal que representa a atividade média de cada região e então calcular a relação estatística entre elas.

Um simples procedimento para se extrair uma rede de conectividade funcional de uma imagem de fMRI consiste dos seguintes passos: (1) os dados devem ser préprocessados para remover ruídos inerentes provenientes de variações fisiológicas ou perturbações na aquisição [22]; (2) parcela-se a imagem em regiões corticais, de acordo com um atlas anatômico pré-definido; (3) Para cara região cortical, é gerada uma série temporal que representa sua atividade, como ilustrado na Figura 1(a) (a série temporal representando a atividade cortical é definida pela média das séries temporais de todos os voxels que delimitam a região cortical definida pelo atlas); (a)

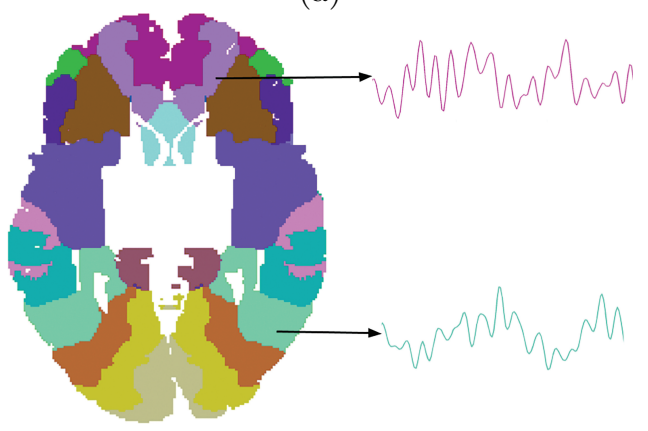

(b)

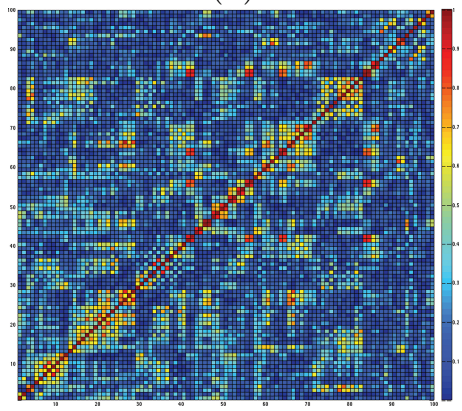

(c)

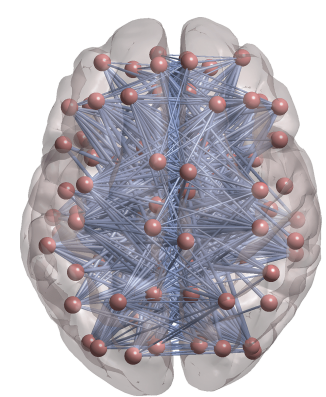

Figura 1: A construção de matrizes de conectividade funcional é feita a partir de: (a) extração de séries temporais (por exemplo, sinal BOLD) de regiões corticais; (b) em seguida, medimos a correlação das séries temporais par a par. Finalmente, (c) obtemos uma representação de rede para o cérebro.

(4) calcula-se a correlação entre as séries das regiões por pares, gerando uma matriz de correlação como representado na Figura 1(b). A matriz de correlação, por mesma, já pode ser considerada uma rede de conectividade funcional, sendo as regiões corticais representadas pelos nós da rede e as correlações pelas conexões, como ilustrado na Figura 11.c). Mais detalhes sobre como definir redes funcionais podem ser encontrados nas referências [23, 25].

No caso em que as conexões possuem pesos diferentes, a rede é dita "ponderada". Se as conexões entre os nós forem simétricas, dizemos que a rede é "não direcionada", ao passo que, quando há uma assimetria nas conexões, a rede é dita "direcionada". Vamos, para ganhar intuição, considerar o exemplo da rede de oficiais em uma organização militar: a relação entre oficiais de diferentes patentes é assimétrica (um sargento obedece à ordens do general, mas não o contrário), portanto essa rede é direcionada; além disso, os pesos das conexões são diferentes, pois, apesar de um soldado obedecer a ambos sargento e general, é o último que possui maior influência sobre o soldado, portanto, a rede é ponderada. Por outro lado, uma idealização de rede de pessoas onde não há senso de superioridade e todos são iguais seria considerada uma rede social não direcionada e não ponderada.

Se o objetivo for analisar a estrutura das conexões, independentemente do peso do valor da correlação, é conveniente transformar as redes em redes não ponderadas. 
Tabela 1: Exemplos de medidas topológicas em redes complexas [69-71].

\begin{tabular}{|c|c|}
\hline Medida topológica & Descrição \\
\hline Grau nodal & $\begin{array}{l}\text { Número de conexões de um nó. O histo- } \\
\text { grama considerando o grau de todos os } \\
\text { nós da rede é chamado de distribuição } \\
\text { de grau. }\end{array}$ \\
\hline Distância geodésica & $\begin{array}{l}\text { Menor caminho entre um par de nós da } \\
\text { rede. }\end{array}$ \\
\hline Coeficiente de aglom & $\begin{array}{l}\text { Fração de primeiros vizinhos de um nó } \\
\text { que são conectados entre si. }\end{array}$ \\
\hline Entropia geodésica & $\begin{array}{l}\text { Medida estatística da diversidades de } \\
\text { influências de um nó. }\end{array}$ \\
\hline Motifs & $\begin{array}{l}\text { Padrões de conectividade que se repe- } \\
\text { tem com considerável probabilidade em } \\
\text { uma rede. }\end{array}$ \\
\hline
\end{tabular}

Isso é feito "binarizando" as matrizes de correlação da seguinte forma: se a correlação for maior que um valor definido, então o elemento de matriz é fixado em 1; caso contrário, em 0 (zero). Isso facilita a detecção de algumas propriedades e torna a rede mais esparsa, facilitando o cálculo computacional para redes com grande número de nós; essa matriz, dita "binária", representa redes não ponderadas e não direcionadas.

A partir das redes funcionais, representadas matematicamente por matrizes, é possível realizar análises topológicas ou dinâmicas e inferir comportamentos locais e globais. Apresentamos algumas métricas topológicas na Tabela 1. Para uma revisão completa sobre métricas de redes, indicamos as referências [26, 27].

Uma hipótese dominante na neurociência contemporânea é de que a estrutura organizacional da conectividade funcional do cérebro humano varia de forma consistente em desordens psíquicas ou em diferentes estados de consciência, sendo corroborada por diversos estudos envolvendo o sono, a meditação ou o estado 
psicodélico 28 33]. Este último será o foco da discussão nas próximas sessões.

\section{Os Psicodélicos}

O estudo contemporâneo das substâncias psicodélicas é marcado por um fato peculiar. Em uma sexta-feira de primavera de 1943, o químico suíço Albert Hofmann foi forçado a interromper seu dia de trabalho após se intoxicar, acidentalmente, com uma substância que havia sintetizado, a dietilamida do ácido lisérgico (LSD) 34. Hofmann reportou o acidente para o diretor do seu laboratório: "[...] uma condição de intoxicação não desagradável, caracterizada por uma imaginação extremamente estimulada" [34]. A síntese do LSD fazia parte de um estudo cujo objetivo era produzir um fármaco que atuasse no sistema respiratório e circulatório - efeitos psíquicos não eram esperados [34]. Esse incidente tornouse um marco histórico para o estudo de substâncias psicodélicas como objeto científico, atraindo também a atenção para outros psicodélicos como a psilocibina e a mescalina, esta sintetizada pela primeira vez em 1919 35.

Os psicodélicos são substâncias serotoninérgicas, isto é, compostos químicos que se ligam aos receptores de serotonina presentes no sistema nervoso central 36. Por fatores ainda pouco conhecidos, eles estimulam o sistema nervoso de forma a causar uma expressiva alteração na qualidade da consciência do indivíduo. As experiências psicodélicas são frequentemente reportadas como místicas ou extraordinárias; os efeitos psíquicos podem incluir despersonalização, recuperação e ressignificação de memórias, potencialização dos sentidos, alucinações, aumento na introspecção e alterações na percepção de espaço e tempo. Em um estudo sobre os efeitos psicológicos de longo prazo decorrentes de uma experiência psicodélica, voluntários foram questionados, dois meses após o evento, o quão significativa a experiência havia sido. Quase $70 \%$ dos voluntários a classificaram como uma das cinco mais importantes de suas vidas [59].

Devido ao conteúdo incomum ao estado de consciência ordinário, as experiências psicodélicas são frequentemente descritas por meio de discurso metafórico, tal como "conexão com o todo", "sentimento oceânico" ou "contato com um deus interior" [37. No livro As Portas da Percepção [38, o escritor britânico Aldous Huxley assim descreveu suas experiências com mescalina em 1953 (grifo no original):

[A] perspectiva se tornara bastante estranha e as paredes da sala já não mais pareciam encontrar-se em ângulos retos. Mas não eram esses os fatos realmente importantes. O que mais ressaltava era a constatação de que as relações espaciais tinham perdido muito do seu valor e de que minha mente tomava contato com o mundo exterior em termos de outras dimensões que não as de espaço. Em situações normais o olho se preocupa com problemas tais como Onde? - A que distância? - Como se situa em relação a tal coisa?. Durante a experiência com a mescalina, as perguntas tácitas a que a visão responde são de outra ordem. Lugar e distância deixam de ter muito interesse. A mente elabora a compreensão das coisas em termos de intensidade de existência, profundidade de importância, relações dentro de um determinado padrão. Eu olhava para os livros, mas não me preocupava, em absoluto, com suas posições no espaço. O que notava, o que se impunha por si mesmo a minha mente, era o fato de que todos eles brilhavam com uma luz viva e que, em alguns, o resplendor era mais intenso que em outros. Nesse instante, a posição e as três dimensões eram questões de somenos. Não, evidentemente, que a noção de espaço houvesse sido abolida. Quando me levantei e pus-me a andar, eu o fiz com toda a naturalidade, sem erros de apreciação sobre a posição dos objetos. O espaço ainda estava ali; mas havia perdido sua primazia. A mente se preocupava, mais do que tudo, não com medidas e lugares, e sim com a existência e o significado.

Em uma série de correspondências com Huxley [39], o psiquiatra britânico Humphry Osmond cunhou o termo "psicodélico" (do grego antigo, psychē, "alma", e dēloun, "revelar") na tentativa de classificar substâncias cujos efeitos não se resumiam simplesmente na mimetização de psicoses (psicotomiméticos). Tomado pela "clareza e eufonia" do termo, Osmond oficializou "psicodélico" nos Anais da Academia de Ciências de Nova York em 1957 [40].

Substâncias psicodélicas são comuns na natureza, podendo ser encontradas em plantas, animais, e até mesmo no corpo humano - em pequenas quantidades [41. O potencial dos psicodélicos em alterar o estado de consciência, possibilitando ao indivíduo acessar conteúdos da mente e explorar conformações de pensamento inéditos ao estado ordinário, encorajou rapidamente psicoterapeutas a considerar o uso dessas substâncias em processos terapêuticos. Apesar de estudos preliminares terem sido feitos na década de 1950 [42, 43], as pesquisas sobre o potencial terapêutico dos psicodélicos ganharam um novo fôlego no início do século XXI 46 51.

A ideia de que o estado de consciência ordinário é somente um dos estados acordados de consciência possíveis, quiçá limitado, ganhou considerável espaço nas décadas posteriores à descoberta de Hofmann. O período histórico era marcado por guerras, tensões diplomáticas, viagens espaciais e grandes revoluções sociais 52 . Movimentos que mais tarde ficaram conhecidos 
como "contracultura" ganharam grande visibilidade e apoio popular. Esses movimentos questionavam valores e padrões sociais vigentes, protestavam contra guerras e privilégios sociais e reivindicavam um estilo de vida alternativo aos padrões da época. Nesse cenário de quebra de paradigmas, as substâncias psicodélicas passam a ganhar significância popular como uma ferramenta progressista, um meio que possibilitava ao indivíduo perceber além dos padrões do contexto em que estava inserido.

Na década de 1970, os psicodélicos passam a ser restritamente regulamentados por um acordo internacional juntamente a dezenas de outros psicoativos [53]. Entre eles fungos, plantas e medicinas tradicionais. Os argumentos que embasaram a "Convenção sobre Substâncias Psicotrópicas" concentraram-se nos potenciais riscos para a saúde e dependência química 54. No entanto, tal medida foi polêmica e ainda hoje fomenta um ardoroso debate. Dentre as críticas, destacamos a generalização de substâncias de natureza completamente distintas, carência (e posteriormente o impedimento) de estudos científicos sobre a natureza e consumo dessas substâncias, e a marginalização de culturas tradicionais. No âmbito epistemológico, a crítica se baseia no direito individual de acessar os conteúdos da própria mente e da realidade que habita.

Apesar de polêmico para a cultura ocidental moderna, culturas antigas já dominavam o uso e as propriedades de psicodélicos. Registros arqueológicos de utensílios com resíduos de substâncias psicodélicas [55, 56] e a presença destas em rituais de culturas tradicionais indicam que o uso de psicodélicos permeia por longo tempo a história da humanidade.

Comunidades tradicionais na América Central faziam uso de cogumelos do gênero Psilocybe (contendo os psicodélicos psilocibina e psilocina) em rituais religiosos [57. Tribos indígenas distintas da região amazônica fazem uso de ayahuasca, uma infusão de plantas contendo $N, N$-dimetiltriptamina (DMT), tal como outras substâncias psicoativas como o rapé, o tabaco e o kambô. A Figura 2 mostra imagens de uma comunidade indígena atual e sua relação com psicoativos. O papel das substâncias psicodélicas nessas comunidades tradicionais não é facilmente compreendida pelos padrões de pensamento cartesiano. Para as tribos indígenas da região amazônica, por exemplo, a ayahuasca tem um papel social, espiritual e medicinal. Chamada por alguns nativos de "medicina sagrada", rituais de curas não diferenciam corpo físico e psíquico. Apesar da incompatibilidade de linguagens, se faz claro que essas culturas possuem grande conhecimento sobre o efeito e propriedades dos psicodélicos em seres humanos.

Atualmente, vivemos a chamada "renascença psicodélica", em que, motivado por trabalhos acadêmicos e clínicos, o interesse por psicodélicos retornou ao debate científico e público, sendo o caráter medicinal um dos principais focos das pesquisas contemporâneas (a)

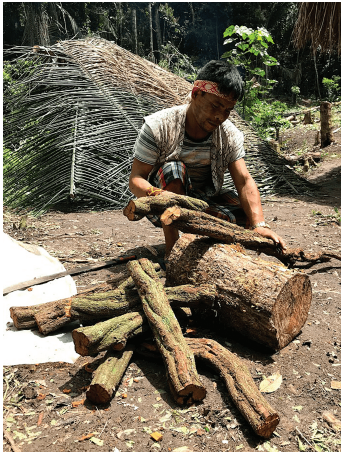

(c)

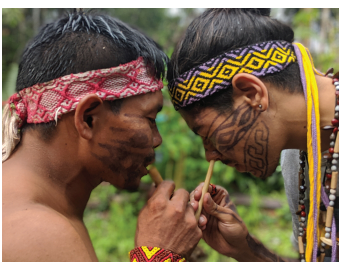

(b)

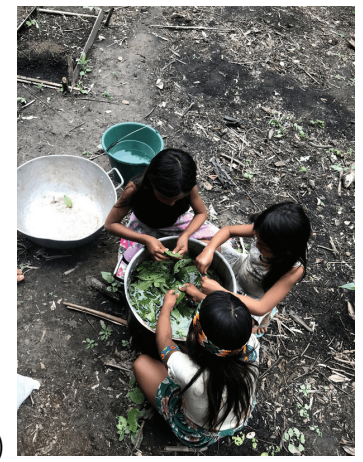

(d)

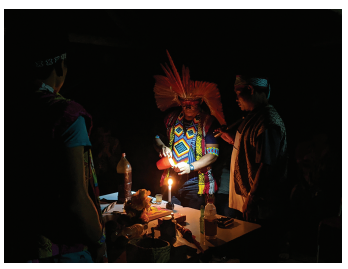

Figura 2: Ayahuasca no contexto indígena. (a) Preparo do Mariri para a ayahuasca: o cipó extraído da floresta é cortado e macerado. (b) Crianças lavam as folhas da chacrona: o preparo do chá envolve toda a comunidade. (c) Passagem de rapé: feito a partir de um pó de tabaco e outras ervas, o rapé está presente em rituais de cura e proteção. (d) Ritual com ayahuasca: a ayahuasca é servida pelo pajé da tribo que conduz a cerimônia.

48, 51, 58. Trabalhos vêm direcionando o foco à pacientes com sintomas de depressão e ansiedade, donde os resultados se mostram promissores [44, 45, 49. Em particular, destacamos um estudo realizado na Universidade Federal do Rio Grande do Norte envolvendo uma única dose de um psicodélico (ayahuasca) administrado a pacientes com depressão resistente (quando não há resposta clínica a diferentes classes de medicamentos). Esse foi o primeiro estudo clínico randomizado e controlado a reportar um rápido efeito antidepressivo de um psicodélico em pacientes com depressão resistente [46].

Nas próximas seções, apresentaremos uma visão panorâmica da ciência sobre psicodélicos, desde o mecanismo de ação dos psicodélicos no receptor de serotonina $5-\mathrm{HT}_{2 \mathrm{~A}}$, à flexibilização de redes funcionais do cérebro humano a partir de métricas da teoria de sistemas complexos. Com base nisso, veremos que a pesquisa com psicodélicos, além do potencial clínico-terapêutico, poderá ter grande valor no entendimento do cérebro humano, possivelmente o mais exemplar e emblemático dos sistemas complexos.

\section{O Cérebro Sob Influência dos Psicodélicos}

A classe de substâncias psicodélicas é definida através de sua ação agonista em receptores de serotonina [36]. Para entender essa definição, vamos lembrar que a serotonina (5-hidroxitriptamina, ou 5-HT) é um neurotransmissor endógeno cuja função biológica é multifacetada, sendo 


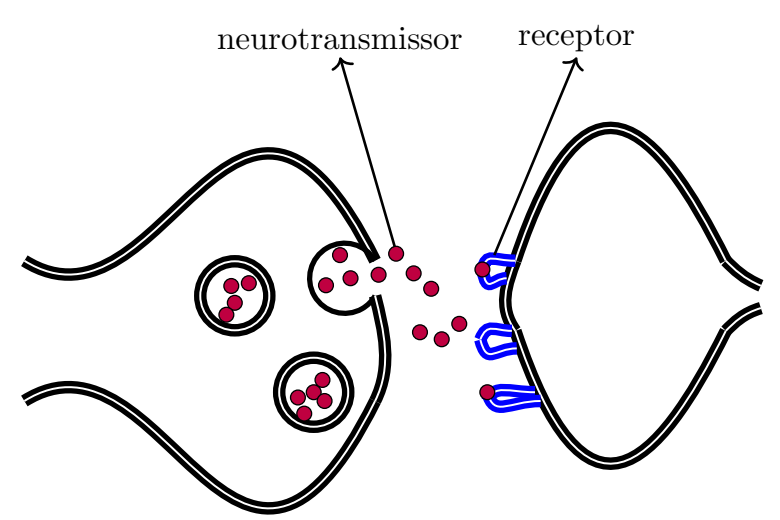

Figura 3: Diagrama simplificado de sinapse química envolvendo a liberação de neurotransmissores por um neurônio pré-sináptico (à esquerda), os quais são captados por receptores na membrana do neurônio pós-sináptico (à direita). Podemos pensar, por exemplo, em serotonina (em púrpuro) ativando os receptores $5-\mathrm{HT}_{2 \mathrm{~A}}$ (em azul).

responsável pela modulação de diversos aspectos fisiológicos e cognitivos, desde humor, apetite, aprendizagem e sono. Essas funções variadas, contudo, têm origem no mesmo processo de liberação de serotonina por neurônios pré-sinápticos, permitindo a transmissão e ação do neurotransmissor em seus respectivos receptores localizados na membrana de neurônios pós-sinápticos (ver Figura 3).

Dizemos que um composto não endógeno tem ação agonista quando sua atuação em receptores acarreta em uma resposta biológica igual ou parcial àquela do neurotransmissor associado. As substâncias psicodélicas, portanto, são definidas a partir de seu agonismo serotoninérgico: elas ativam (estimulam) os receptores de serotonina, produzindo uma resposta biológica similar à da serotonina (ver Figura 4). Já se mostrou que a afinidade de substâncias psicodélicas para com o receptor $5-\mathrm{HT}_{2 \mathrm{~A}}$ é fortemente correlacionada com os efeitos psicológicos em humanos 60 62, mas não sabemos se a ativação do $5-\mathrm{HT}_{2 \mathrm{~A}}$ é condição suficiente para a ocorrência dos efeitos, haja visto que os psicodélicos também atuam em outros receptores de serotonina e dopamina. Nota-se que, já na escala de neurotransmissores, cérebro apresenta um desafiador problema de modelagem. Todavia, a partir de agora trataremos da influência de psicodélicos sobre populações de neurônios e redes funcionais.

Localizando-se principalmente em corpos celulares e dendritos de neurônios (especificamente, neurônios piramidais da camada $\mathrm{V}$ do córtex cerebral), os receptores $5-\mathrm{HT}_{2 \mathrm{~A}}$ são densamente distribuídos em regiões corticais do sistema visual 63, possivelmente sendo responsáveis pelos proeminentes efeitos visuais da experiência psicodélica. Além disso, os receptores $5-\mathrm{HT}_{2 \mathrm{~A}}$ são expressos em regiões de alto nível funcional, como aquelas constituindo a rede de modo padrão (DMN, do inglês, Default Mode Network) [64, uma rede cerebral de larga escala (ver Seção II) associada a processos mentais (a) Serotonina (5-HT)

(b) LSD
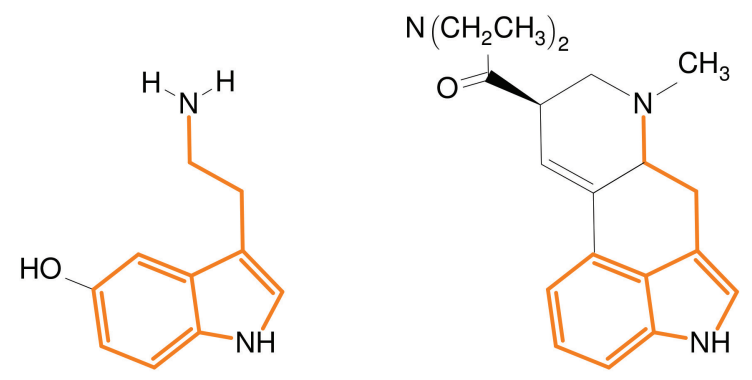

(c) Psilocibina $\left(\mathrm{R}=\mathrm{PO}_{3} \mathrm{H}^{-}\right)$

(d) DMT
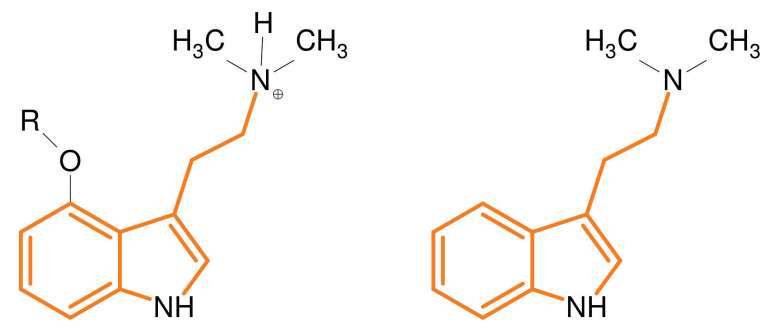

Figura 4: Estrutura molecular do neurotransmissor (a) serotonina, e dos psicodélicos (b) LSD, (c) psilocibina $\left(\mathrm{R}=\mathrm{PO}_{3} \mathrm{H}^{-}\right)$ ou psilocina $(\mathrm{R}=\mathrm{H})$, e (d) DMT. Em laranja, destacamos o esqueleto triptamínico comum a todas as quatro moléculas e indicativo de que os psicodélicos tenham afinidade serotoninérgica.

internamente orientados, como a divagação, e o "sonhar acordado"; de fato, a DMN se mostra ativa em condições de repouso desperto, contrastando-se das situações de desativação envolvendo a execução de tarefas cognitivas externamente orientadas.

Uma série de condições psiquiátricas e estados de consciência já foram associados à atividade intrínseca da DMN; por exemplo, o seu aumento de atividade foi constatado na esquizofrenia [65] e depressão 66], ao passo que a doença de Alzheimer e estados meditativos apresentaram redução na atividade da DMN [67, 68. Em particular, durante seus efeitos agudos, os psicodélicos consistentemente se mostraram reduzindo a atividade e conectividade na DMN, causando desagregação e integração global de redes funcionais 32 .

Para se entender esse fenômeno, lançou-se mão de um modelo combinando conceitos de teoria da informação e física estatística aplicada às ciências cognitivas, a hipótese do cérebro entrópico, a qual discutiremos na próxima seção.

\section{A Hipótese do Cérebro Entrópico}

Em física estatística, entropia de Boltzmann é uma grandeza que mede a quantidade de microestados 
compatíveis com um determinado macroestado de um sistema físico; grosso modo, é uma forma de contar o número de arranjos microscópicos admitidos por uma certa configuração macroscópica de um sistema. Se há muitas maneiras de rearranjar-se a configuração microscópica de um sistema sem que isso mude sua configuração macroscópica, então o sistema é dito altamente entrópico; do contrário, havendo poucos microestados compatíveis com um dado macroestado, então o sistema possui baixa entropia.

Tomemos como exemplo o processo irreversível de misturar café com leite. Considere, inicialmente, meio copo de café e outro meio copo de leite em copos separados. Seja $\Omega_{l}$ e $\Omega_{c}$ as estimativas para os números de microestados do leite e do café, respectivamente, tal que o número total de configurações seja $\Omega_{l} \Omega_{c}$. A entropia total então será dada pela fórmula de Boltzmann, $S=$ $k_{B} \log \left(\Omega_{l} \Omega_{c}\right)$. Por outro lado, se o café e leite ficarem misturados em um único copo, o número de microestados será muito maior do que $\Omega_{l} \Omega_{c}$. Consequentemente, a entropia da mistura também será maior do que para o café e leite tratados separadamente. Esse aumento é conhecido como entropia de mistura. É esse aumento de entropia que faz com que seja difícil "desmisturar" algo que já foi misturado.

Não obstante, no contexto de teoria da informação, entropia também pode ser entendida como uma medida de incerteza associada a um sistema; um dado viciado, por exemplo, possui menor entropia de Boltzmann-Gibbs comparado a um dado normal com o mesmo número de faces, pois os resultados de lançamento do primeiro são menos incertos que o segundo. As diversas formas de entropia, sejam inspiradas pela física estatística ou pela teoria de informação, têm motivado estudos e hipóteses nas ciências cognitivas.

Devido à dificuldade, ou até mesmo impossibilidade, de quantificar a qualidade de conteúdos mentais, não há ainda estudos pragmáticos calculando a entropia de estados mentais. No entanto, o aumento na imprevisibilidade e diversidade dos conteúdos mentais durante experiências psicodélicas se faz claro em relatos de experiências, textos, pinturas, entre outros. Na tentativa de explicar os mecanismos por detrás desse aumento na diversidade do estado mental do indivíduo sob influência de psicodélicos, Carhart-Harris e seus colaboradores levantaram uma hipótese, denominada "hipótese do cérebro entrópico", que diz respeito ao uso do conceito de entropia como forma de quantificar a variedade de estados mentais considerando medidas de entropias na atividade cerebral [31.

Essa hipótese sugere que entropias de grandezas relacionadas aos aspectos funcionais da atividade cerebral (por exemplo, séries temporais de neuroimagem) poderiam permitir distinguir quantitativamente as diferenças entre os diversos estados subjetivos, sejam estes patológicos ou saudáveis. A hipótese do cérebro entrópico, portanto, prevê diferentes estados mentais apresentando diferentes assinaturas entrópicas. Em particular, ela implica que os estados de consciência induzidos por substâncias psicodélicas apresentam maior entropia quando comparados à entropia de estados ordinários e depressivos.

Para encerrar esta seção, podemos assim resumir a hipótese do cérebro entrópico: os efeitos das substâncias psicodélicas seriam causados pela estimulação de receptores $5-\mathrm{HT}_{2 \mathrm{~A}}$ distribuídos pelo córtex cerebral, despolarizando neurônios piramidais e dessincronizando a atividade cortical. A partir disso, uma desintegração e desagregação de redes cerebrais de grande escala se estabeleceria no cérebro, manifestando-se, finalmente, em parâmetros funcionais cujas distribuições de probabilidade poderiam ser estudadas valendo-se da entropia das mesmas [31. Estas revelariam um repertório maior de padrões funcionais, refletindo, por fim, no aumento na entropia do cérebro.

\section{Física Estatística Aplicada ao Estudo de Psicodélicos}

Para finalizar, revisamos alguns estudos em que ferramentas e conceitos de física estatística foram usados para avaliar dados de neuroimagem de indivíduos sob influência de psicodélicos. Em especial, resumimos resultados de um estudo feito com indivíduos sob efeito da decocção psicodélica ayahuasca. Detalhes desse estudo podem ser encontrados nas referências [21, 171.

Proveniente da cultura indígena da América do Sul, a ayahuasca é extraída da mistura de duas plantas originárias da região amazônica: o arbusto chacrona, que contém a substância psicodélica DMT, e o cipó mariri, ou huasca, que contém inibidores da enzima monoamina oxidase. Esta última evita a rápida degradação do DMT no sistema digestivo, permitindo que a substância penetre a barreira hematoencefálica e provoque efeitos psicoativos. Para mais informações sobre ayahuasca, indicamos as referências $[72-74$.

Voluntários foram submetidos a uma sessão de neuroimageamento cerebral por fMRI em estado ordinário de consciência, ou seja, antes de ingerir a dose de ayahuasca; e outra sessão 40 minutos após a ingestão do chá, quando os efeitos psíquicos atingem um pico de intensidade. Em ambas sessões, os voluntários permaneceram em estado de repouso desperto, o qual consiste em permanecer deitado, imóvel, com os olhos fechados e sem realizar nenhuma tarefa. Nesse estudo, as imagens de fMRI foram inicialmente pré-processadas e, posteriormente, geraram-se redes funcionais como descrito na Seção II. Os dados a serem avaliados consistiram de um conjunto de redes não ponderadas e com diferentes densidades de conexões para cada uma das amostras, ambos antes e depois de ingerir ayahuasca.

As redes funcionais cerebrais referentes aos indivíduos sob influência de ayahuasca apresentaram variações locais e globais consistentes para todos os indivíduos. Em particular, as redes funcionais depois da ingestão 
do chá apresentam um maior coeficiente de aglomeração e uma maior eficiência local, indicando que, na média, as redes são localmente mais conectadas. O contrário ocorre a nível global: na média, o menor caminho médio aumenta, e a eficiência global da rede diminui, indicando que as redes se tornam menos globalmente integradas. Uma observação intrigante: a redução na integração da rede é positivamente correlacionada com os níveis de estados dissociativos avaliado por uma escala psicométrica [75]. Estados dissociativos podem corresponder ao sentimento "oceânico" e "dissolução do ego" frequentemente reportados em experiências psicodélicas. É importante ressaltar que o número muito pequeno de indivíduos nesse estudo não permite conclusões quanto a essa observação, no entanto, esses resultados apontam para a necessidade de estudos mais aprofundado sobre essa relação.

Medidas de entropia baseadas na teoria da informação indicam que as redes funcionais de indivíduos sob influência de ayahuasca são menos constritas. A entropia da distribuição de grau aumenta, indicando que há uma diversidade maior no grau de conectividade dos nós - existem mais nós com alto grau e mais nós com baixo grau, sugerindo uma quebra da hierarquia da rede. A entropia geodésica, a qual quantifica a complexidade da arquitetura da rede avaliada do ponto de vista de cada nó, também aumenta [71]. Ou seja, sob a influência de psicodélicos, há aumento da entropia relativa à estrutura de correlações a qual uma dada região cortical está submetida.

Dado os resultados obtidos para a entropia durante o estado psicodélico, poderíamos nos perguntar: estaria o aumento na entropia das redes funcionais relacionado ao aumento da "entropia dos conteúdo mentais"? A entropia em redes funcionais cerebrais foram investigadas também em outros trabalhos com diferentes abordagens [76] 78. Em um estudo com indivíduos sob efeito de psilocibina, a estatística foi realizada considerando-se a diversidade de motifs na rede, que são definidos como padrões de sub-redes repetindo-se dentro da rede; mostrou-se que, sob efeito de psilocibina, a entropia de motifs aumenta [78. É possível que o aumento de entropia nas redes funcionais seja possibilitado pela redução em padrões de hierarquia na rede. Estudos sugerem que as redes do cérebro humano possuem organização hierárquica e modular, as quais apresentam divisões em subgrupos de nós, ou módulos, em que as conexões são mais densas dentro dos módulos do que entre os módulos. Essas características podem ser a ponte entre estudos de entropia e de criticalidade em redes.

A flexibilidade e a capacidade de armazenamento do cérebro humano sugerem que este funciona em um regime crítico ou similar [79, 80. O regime crítico se caracteriza por um decaimento lento nas correlações espaciais e temporais. Tipicamente, o sistema apresenta propriedades livre de escala: as flutuações são mais imprevisíveis, embora não aleatórias; e o sistema possui um repertório maior de estados do que quando se encontra longe da criticalidade. Estudos teóricos têm evidenciado que esse regime pode surgir em redes hierárquicas modulares [81, 82] ou mesmo em redes somente modulares [83]. Uma possível interpretação sugere que a quebra da hierarquia, bem como a variação nas características modulares nas redes funcionais de indivíduos sob efeito de psicodélicos, sejam responsáveis por modificar aspectos de criticalidade relacionados à flexibilidade dos estados mentais. O estudo direto de criticalidade em redes funcionais de larga escala é limitado devido ao reduzido tamanho das redes. No entanto, o aumento nas entropias das redes funcionais pode estar refletindo uma quebra de hierarquia. A isso, uma hipótese plausível afirma que a quebra da hierarquia, resultando em uma variação no comportamento crítico, direta e completamente explica o aumento na flexibilidade dos estados mentais. Outra hipótese é que, em indivíduos sob efeito de psicodélicos, cada região cortical poderia funcionar mais livremente, com menos influência das demais, ou então mais livre e sob menos controle de uma possível estrutura central.

É importante destacar que, dentre as características de sistemas complexos mencionadas na introdução deste artigo, abordamos somente os aspectos relacionados à emergência e às correlações. Todavia, nos perguntamos se é possível avançar no entendimento dos efeitos de psicodélicos investigando-se algumas das outras características de sistemas complexos, tais como organização hierárquica, dinâmicas e relacionamentos não lineares, auto-organização. Esse é um problema em aberto.

Apesar de grandes avanços, as teorias de como o cérebro funciona, sob a ótica da física estatística, são ainda preliminares. Hoje, a física estatística aplicada à neurociência dos psicodélicos se concentra, em maior parte, no estudo de dados, como os obtidos por neuroimagem e escalas psicométricas. Temos, atualmente, ambas quantidades e qualidade de dados inéditas para o estudo não somente do efeito de psicodélicos, mas para o estudo do cérebro humano em geral. A variabilidade das características das redes cerebrais funcionais dentre diferentes indivíduos é ampla, e sua unicidade pode ser comparada com a unicidade de impressões digitais. Essa variabilidade aumenta sob influência de psicodélicos. $\mathrm{O}$ sucesso em caracterizar e diferenciar redes funcionais de indivíduos em estado ordinário de consciência e estados psicodélicos usando ferramentas, conceitos e interpretações da física estatística concede considerável credibilidade a essa abordagem. Em suma, o estudo de psicodélicos sob essa perspectiva é um campo novo e promissor, aberto a inúmeras possibilidades.

\section{Comentários Finais}

O estudo do cérebro humano tem avançado consideravelmente por meio da aplicação de métodos de física estatística nas ciências cognitivas. Em concordância, vimos que o cérebro é um exemplar sistema dinâmico, 
apresentando características de complexidade desde os seus neurônios às redes de conectividade funcional, tornando evidente uma necessária colaboração entre a física e neurociência.

Valendo-se dessa colaboração, trouxemos à luz uma recente e promissora área de investigação: a influência de substâncias psicodélicas no cérebro humano. Destacamos que, nos últimos anos, a redescoberta do potencial terapêutico de psicodélicos no tratamento de transtornos de humor (por exemplo, depressão) fomentou novos modelos da cognição humana, tal como a hipótese do cérebro entrópico, a qual propõe um aumento de entropia associado aos correlatos neurais da experiência psicodélica. De fato, a hipótese do cérebro entrópico tem sido corroborada por diversos trabalhos desde a sua concepção; o cérebro sob influência de psicodélicos aparenta operar em um estado de maior entropia, manifestando-se, por exemplo, em termos de redes funcionais menos constritas, possivelmente servindo como base terapêutica na flexibilização de estados mentais ruminantes, característicos da depressão e outros transtornos.

Iniciada há poucos anos, a "renascença psicodélica" é a continuação de um processo interrompido no conturbado período político da década de 1960. Esperamos que, além de ilustrar as atuais e promissoras técnicas do estudo da complexidade cerebral, este trabalho também possa ter evidenciado o papel essencial da ciência em participar do debate público e influenciar décadas do rumo da sociedade.

\section{Agradecimentos}

Agradecemos ao CNPq (processo 302051/2018-0) à CAPES pelo apoio financeiro. AV agradece o projeto PRIN grant 20174TPEFJ, "TRIPS", pelo suporte e A. Treves por discussões. Agradecemos Jonathan Joabe e a tribo Huni Kuin pelas fotografias.

\section{Referências}

[1] K. Mainzer, Thinking in Complexity: The Complex Dynamics of Matter, Mind, and Mankind (Springer-Verlag Berlin Heidelberg, Heidelberg, 2013).

[2] Y. Bar-yam, Dynamics Of Complex Systems (CRC Press, Boca Raton, 2019).

[3] H.J. Morowitz, The Mind, The Brain And Complex Adaptive Systems (Routledge, New York, 2018).

[4] M. Li e P. Vitanyi, An Introduction to Kolmogorov Complexity and Its Applications (Springer, New York, 2013).

[5] H.E. Stanley, L.A.N. Amaral, S.V. Buldyrev, A.L. Goldberger, S. Havlina, H. Leschhorn, P. Maass, H.A. Makse, C.K. Peng, M.A. Salinger et al., Physica A 231, 20 (1996).

[6] H.E. Stanley, V. Afanasyev, L.A.N. Amaral, S.V. Buldyrev, A.L. Goldberger, S. Havlina, H. Leschhorn, P. Maass, R.N. Mantegna, C.K. Peng et al., Physica A 224, $302(1996)$.
[7] T. Tomé, Tendências da física estatística no Brasil: homenagem ao professor Silvio Salinas no seu $60^{\circ}$ aniversário (Editora Livraria da Física, São Paulo, 2003).

[8] I. Gleria, R. Matsushita e S. Da Silva, Rev. Bras. Ensino Fís. 26, 99 (2004).

[9] S. Salinas, Introduction to Statistical Physics (Springer, New York, 2001).

[10] H.E. Stanley, Introduction to Phase Transitions and Critical Phenomena (Oxford University Press, Oxford, 1987).

[11] J. Hesse e T. Gross, Front. Syst. Neurosci. 8, 166 (2014).

[12] S. Herculano-Houzel, Front. Hum. Neurosci. 3, 31 (2009).

[13] K. Im, J.M. Lee, U. Yoon, Y.W. Shin, S.B. Hong, I.Y. Kim, J.S. Kwon e S.I. Kim, Hum. Brain Mapp. 27, 994 (2006).

[14] L. Tuominen, L. Nummenmaa, L. Keltikangas-Järvinen, O. Raitakari e J. Hietala, Hum. Brain Mapp. 35, 1875 (2014).

[15] O. Sporns, Ann. NY Acad. Sci. 1224, 109 (2011).

[16] L. Brochini, A.A. Costa, M. Abadi, A.C. Roque, J. Stolfi e O. Kinouchi, Sci. Rep. 6, 35831 (2016).

[17] C.W. Lynn e D.S. Bassett, Nat. Rev. Phys. 1, 318 (2019).

[18] C.M. Michel e D. Brunet, Front. Neurosci. 10, 325 (2019).

[19] R.B. Buxton, Rep. Prog. Phys. 76, 96601 (2013).

[20] R. Covolan, D.B. de Araújo, A.C. dos Santos e F. Cendes, Ciência e Cultura 56, 40 (2004).

[21] A. Viol, Entropia de Shannon e propriedades topológicas de redes funcionais do cérebro humano sob efeito de ayahuasca. Tese de Doutorado, Universidade Federal do Rio Grande do Norte, Natal (2015).

[22] W.R. Shirer, H. Jiang, C.M. Price, N. Bernard e M.D. Greicius, Neuroimage 117, 1053 (2015).

[23] F.D.V. Fallani, J. Richiardi, M. Chavez e S. Achard, Philos. Trans. Royal Soc. 369, 20130521 (2014).

[24] H. Onias, A. Viol, F. Palhano-Fontes, K.C. Andrade, M. Sturzbecher, G. Viswanathan e D.B. de Araujo, Epilepsy Behav. 38, 71 (2014).

[25] O. Sporns, Dialogues Clin. Neurosci. 15, 247 (2013)

[26] A. Barrat, M. Barthélemy e A. Vespignani, Dynamical Processes on Complex Networks (Cambridge University Press, New York, 2008).

[27] A. Fornito, A. Zalesky e E. Bullmore, Fundamentals of Brain Network Analysis (Academic Press, Cambridge, 2016).

[28] M. Lynall, D.S. Bassett, R. Kerwin, P.J. McKenna, M. Kitzbichler, U. Muller e E. Bullmore, J. Neurosci. 30, 9477 (2010)

[29] F.A. Cotier, R. Zhang e T.M.C. Lee, Sci. Rep. 7, 598 (2017)

[30] A. Kazeminejad e R.C. Sotero, Front. Neurosci. 12, 1018 (2019).

[31] R.L. Carhart-Harris, D. Erritzoe, T. Williams, J.M. Stone, L.J. Reed, A. Colasanti, R.J. Tyacke, R. Leech, A.L. Malizia, K. Murphy et al., Proc. Natl. Acad. Sci. U.S.A. 109, 2138 (2012).

[32] F. Palhano-Fontes, K.C. Andrade, L.F. Tofoli, A.C. Santos, J.A.S. Crippa, J.E.C. Hallak, S. Ribeiro e D.B. de Araujo, PLoS One 10, e0118143 (2015). 
[33] A.B.A. Stevner, D. Vidaurre, J. Cabral, K. Rapuano, S.F.V. Nielsen, E. Tagliazucchi, H. Laufs, P. Vuust, G. Deco, M.W. Woolrich et al., Nat. Commun. 10, 1035 (2019).

[34] A. Hofmann, LSD, My Problem Child: Reflections on Sacred Drugs, Mysticism, and Science (Multidisciplinary Association for Psychedelic Studies, Santa Cruz, 2005).

[35] M. Jay, Mescaline: A Global History of the First Psychedelic (Yale University Press, Londres, 2019).

[36] D.E. Nichols, Pharmacol. Rev. 68, 264 (2016).

[37] S. Grof, LSD Psychotherapy (Hunter House, Pomona, 1980).

[38] A. Huxley, As Portas da Percepção (Editora Globo, São Paulo, 2002).

[39] C.C. Bisbee, P. Bisbee, E. Dyck e P. Farrell, Psychedelic Prophets: The Letters of Aldous Huxley and Humphry Osmond (McGill-Queen's University Press, Montreal, 2018).

[40] H. Osmond, Ann. NY Acad. Sci. 66, 418 (1957).

[41] S.A. Barker, Front. in Neuro. 12, 536 (2018).

[42] T.S. Krebs e P.Ø. Johansen, J. Psychopharmacol. 26, 99 (2012).

[43] J.J. Rucker, L.A. Jelen, S. Flynn, K.D. Frowde e A.H. Young, J. Psychopharmacol. 30, 1220 (2016).

[44] F.L. Osório, R.F. Sanches, L.R. Macedo , R.G. Santos, J.P. Maia-de-Oliveira, L. Wichert-Ana, D.B. Araujo, J. Riba, J.A. Crippa e J.E. Hallak, Braz J Psychiatry. 37, 13 (2015).

[45] R.L. Carhart-Harris, L. Roseman, M. Bolstridge, L. Demetriou, J.N. Pannekoek, M.B. Wall, M. Tanner, M. Kaelen, J. McGonigle, K. Murphy et al., Sc. Rep. 7, 2045 (2017).

[46] F. Palhano-Fontes, D. Barreto, H. Onias, K.C. Andrade, M.M. Novaes, J.A. Pessoa, S.A. Mota-Rolim, F.L. Osório, R. Sanches, R.G. dos Santos et al., Psychol. Med. 49, 655 (2019).

[47] F. Palhano-Fontes, Os efeitos antidepressivos da ayahuasca, suas bases neurais e relação com a experiência psicodélica. Tese de Doutorado, Universidade Federal do Rio Grande do Norte, Natal (2017).

[48] E.E. Schenberg, Braz. J. of Psychiatry (2020), unpublished.

[49] R.G. dos Santos, F.L. Osório, J.A.S. Crippa, J. Riba, A.W. Zuardi e J.E.C. Hallak, Ther. Adv. Psychopharmacol. 6, 193 (2016).

[50] L. Roseman, L. Demetriou, M.B. Wall, D.J. Nutt e R.L. Carhart-Harris, Neuropharmacol. 142, 263 (2018).

[51] B. Labate e C. Cavnar. The Therapeutic Use of Ayahuasca (Springer-Verlag Berlin Heidelberg, Heidelberg, 2014).

[52] E.J. Hobsbawm e M. Santarrita, Era dos extremos: o breve século XX, 1914-1991 (Companhia das Letras, São Paulo, 1995).

[53] D.R. Bewley-Taylor, Int. J. Drug Policy 14, 171 (2003).

[54] United Nations Office on Drugs and Crime, The international drug control conventions, United Nations, Viena (2013).

[55] H.R. El-Seedi, P.A. De Smet, O. Beck, G. Possnert e J.G. Bruhn, J. Ethnopharmacol. 101, 238 (2005).
[56] M.J. Miller, J. Albarracin-Jordan, C. Moore e J.M. Capriles, Proc. Natl. Acad. Sci. U.S.A. 116, 11207 (2019).

[57] R.E. Schultes e A. Hofmann, Plants of the Gods: Origins of Hallucinogenic Use (Alfred van der Marck Editions, New York, 1979).

[58] F.S. Barrett, M.K. Doss, N.D. Sepeda, J.J. Pekar e R.R. Griffiths, Sci. Rep. 10, 2214 (2020).

[59] R.R. Griffiths, W.A. Richards, U. McCann e R. Jesse, Psychopharmacol. 187, 268 (2006).

[60] F.X. Vollenweider, M.F. Vollenweider-Scherpenhuyzen, A. Bäbler, H. Vogel e D. Hell, Neuroreport. 9, 3897 (1998).

[61] R.A. Glennon, M. Teitler e E. Sanders-Bush, NIDA Res. Monogr. 119, 131 (1992).

[62] D.A. Martin e C.D. Nichols, EBioMedicine 11, 262 (2016).

[63] A.W. Moreau, M. Amar, N. Le Roux, N. Morel e P. Fossier, Cereb. Cortex 20, 456 (2010).

[64] R.L. Carhart-Harris e K.J. Friston, Pharmacol. Rev. 71, 316 (2019).

[65] A.G. Garrity, G.D. Pearlson, K. McKiernan, D. Lloyd, K.A. Kiehl e V.D. Calhoun, Am. J. Psychiatry 164, 450 (2007).

[66] Y.I. Sheline, D.M. Barch, J.L. Price, M.M. Rundle, S.N. Vaishnavi, A.Z. Snyder, M.A. Mintun, S. Wang, R.S. Coalson e M.E. Raichle, Proc. Natl. Acad. Sci. U.S.A. 106, 1942 (2009).

[67] M.D. Greicius, G. Srivastava, A.L. Reiss e V. Menon, Proc. Natl. Acad. Sci. U.S.A. 101, 4637 (2004).

[68] J.A. Brewer, P.D. Worhunsky, J.R. Gray, Y.Y. Tang, J. Weber e H. Kober, Proc. Natl. Acad. Sci. U.S.A. 108, 20254 (2011).

[69] M. Newman, Networks: An Introduction (OUP Oxford, Oxford, 2010).

[70] A.L. Barabási e M. Pósfai, Network Sciences (Cambridge University Press, Cambridge, 2016).

[71] A. Viol, F. Palhano-Fontes, H. Onias, D.B. de Araujo, P. Hövel e G.M. Viswanathan, Entropy 21, 128 (2019).

[72] B. Labate e C. Cavnar. Ayahuasca Shamanism in the Amazon and Beyond (Oxford University Press, Oxford, 2014).

[73] B. Labate e C. Cavnar. The Antipodes of the Mind: Charting the Phenomenology of the Ayahuasca Experience (Oxford University Press, Oxford, 2003).

[74] J. Riba, A. Rodriguez-Fornells, G. Urbano, A. Morte, R. Antonijoan, M. Montero, J.C. Callaway e M.J. Barbanoj, Psychopharmacol. 154, 85 (2001).

[75] J.D. Bremner, J.H. Krystal, F.W. Putnam, S.M. Southwick, C. Marmar, D.S. Charney e C.M. Mazure, J. Trauma. Stress 11, 125 (1998).

[76] M.M. Schartner, R.L. Carhart-Harris, A.B. Barrett, A.K. Seth e S.D. Muthukumaraswamy, Sci. Rep. 7, 46421 (2017).

[77] A.V. Lebedev, M. Kaelen, M. Lovden, J. Nilsson, A. Feilding, D.J. Nutt e R.L. Carhart-Harris, Hum. Brain Mapp. 9, 3203 (2016).

[78] E. Tagliazucchi, R. Carhart-Harris, R. Leech, D. Nutt e D.R. Chialvo, Hum. Brain Mapp. 35, 5442 (2014). 
[79] J.M. Beggs e D. Plenz, J. Neurosci. 23, 11167 (2003).

[80] A.J. Fontenele, N.A.P. de Vasconcelos, T. Feliciano, L.A.A. Aguiar, C. Soares-Cunha, B. Coimbra, L. Dalla Porta, S. Ribeiro, A.J. Rodrigues e N. Sousa, Phys. Rev. Lett. 122, 208101 (2019).

[81] G. Ódor, R. Dickman e G. Ódor, Sci. Rep. 5, 14451 (2015).

[82] P. Moretti e M. Muñoz, Nat. Commun., 4, 2521 (2013).

[83] W. Cota, G. Ódor e S.C. Ferreira, Sci. Rep. 8, 9144 (2018). 